\title{
Reduce Charging Effects on Beam Sensitive Material by Optimized Scanning Routines in SEM
}

\author{
Maximilian Schmid ${ }^{1 *}$, Andreas Liebel ${ }^{1}$, Friederike Michael ${ }^{1}$, Grigore Moldovan ${ }^{2}$, and Heike Soltau ${ }^{1}$ \\ 1. PNDetector GmbH, Otto-Hahn-Ring 6, 81739 München, Germany \\ 2. point electronic GmbH, Erich-Neuß-Weg 15, 06120 Halle (Saale), Germany) \\ * Corresponding author: Maximilian.Schmid@pndetector.de
}

Today's electron microscopes enable sample imaging and inspection with low electron energies at a high level of resolution and scan speed with great stability and reliability. This enables the examination of beam sensitive samples like biological or insulating specimens. Charging effects on specimens with poor conductivity can obscure images by distortion or artificial contrast. In case of BSE imaging, this can lead to misinterpretations in compositional examinations. In this paper, we show how high scan speeds and large area imaging with high pixel count can help to reduce these charging effects. The standard procedure to reduce charging is to lower the beam energy or current and to use, if possible, low vacuum modes or discharging devices. However, next to these, the method of scanning the sample can help to further reduce charging and sample damage. This method can be divided into two stages. First, we show how faster scan speeds reduce charging, and second, how larger scan areas with high pixel counts can help to further improve results.

As sketched in fig. 1(c), the shorter the time the beam rests on each pixel (i.e. the pixel dwell time ( $\left.t_{d w e l l}\right)$ the less charge builds up and the faster the pixel will be discharged. This reduces damage and artificial contrast. Fig. 1(a-b) shows a comparison of two images of silver flakes in an organic matrix. Both micrographs are taken at the same brightness and contrast settings and all SEM parameters where kept the same except scan speed and frame averaging. Fig. 1(a) is taken with $t_{\text {dwell }}=400$ ns and zero frame average while fig. 1(b) is taken with $t_{d w e l l}=40 \mathrm{~ns}$ and 10 frame averaging, which results in the same electron dose. It is clearly visible that the faster scan speed reduce the charging effects. The artificial contrast between the silver flakes in the highlighted section 1 and 2 is reduced as well as the overall charging of the organic matrix. Focusing again on the charging of a single pixel, fig. 1(d) sketches repeated scans. There are mainly two cases. Either the pixel discharges until the beam passes the pixel for the next time or the continuous scans will accumulate charges. To remove this charge accumulation the time between beam passes needs to be elongated. This can be managed by an interframe waiting time.

When considering large area scans, which are often performed by stitching methods there is another way to overcome the inter-frame waiting time. By enlarging the scan area and the pixel count by the same factor, the pixel size is kept constant but the time between two passes of the electron beam can be significantly increased. Fig. 2(a) shows a 1 Mpixel cutout of a 100 Mpixel, $500 \mu \mathrm{m}$ square image taken again at $\mathrm{t}_{\mathrm{dwell}}=40 \mathrm{~ns}$ with 10 frame average. Compared to the $1 \mathrm{Mpixel}$ image in fig 1(b), where the beam passes each pixel roughly every $6 \mathrm{~ms}$ (including flyback times, etc.) this time increases to about $6 \mathrm{~s}$ in the case of $100 \mathrm{Mpixel}$ image. This gives each pixel a 1000 times longer period to discharge, resulting in a further reduction of charging effects. Fig 2(c) shows a comparison of the artificial Z-contrast of two silver flakes in the highlighted areas of fig. 1(a-b) and fig. 2(a), nearly vanishing in the latter case. Besides presenting the optimized scanning routines this talk will focus on how they can be applied to beam sensitive biological and semiconductor samples. Further examples will be presented. 

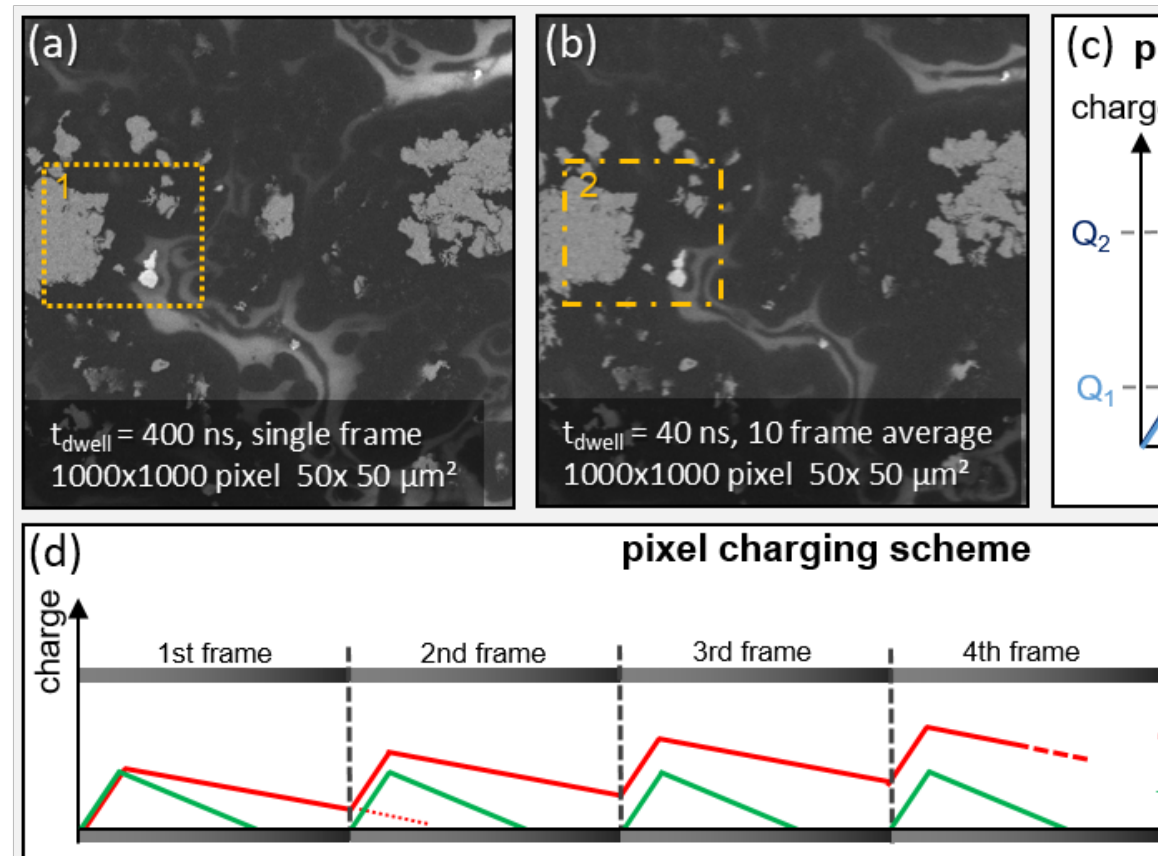
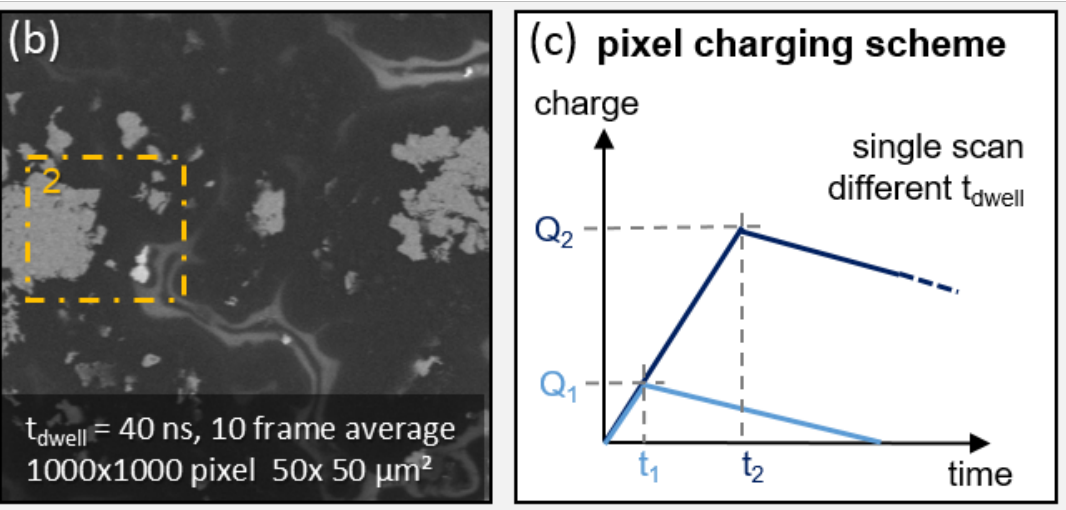

continuous scan

different discharging

constants

charge accumulation

fully discharged

time

Figure 1. (a-b) BSE image of silver flakes in an organic matrix. Images taken with: $E_{\text {beam }}=3 \mathrm{keV}$, $\mathrm{I}_{\text {beam }}=120 \mathrm{pA}$, resolution 1 Mpixel, $50 \mu \mathrm{m}$ edge length, (a) $\mathrm{t}_{\text {dwell }}=400 \mathrm{~ns}$, single frame, (b) $t_{d w e l l}=40$ ns, 10 frame average; (c-d) Schematic illustration of the charging of a single pixel for (a) single beam pass and (b) continuous scanning.

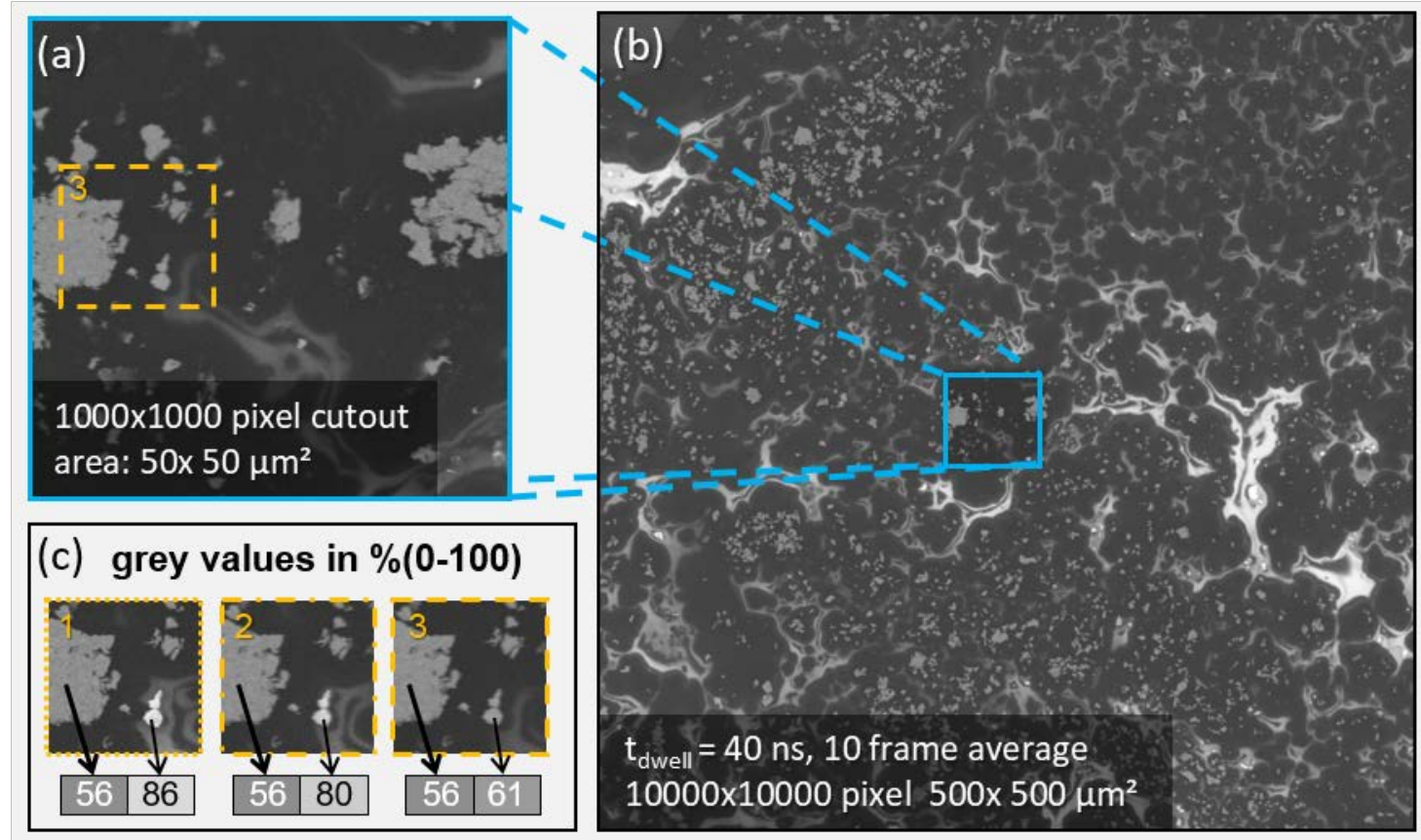

Figure 2. (a) 1 Mpixel coutout of (b) with $50 \mu \mathrm{m}$ edge length equal to fig. 1(a-b); (b) BSE image of silver flakes in an organic matrix. Image taken with: Ebeam $=3 \mathrm{keV}$, Ibeam $=120 \mathrm{pA}$, resolution 100 Mpixel, $500 \mu \mathrm{m}$ edge length, $t_{d w e l l}=40 \mathrm{ns,} 10$ frame average; (c) Comparison of the artificial contrast between two silver flakes highlighted in fig. 1(a-b) and fig. 2(a) 